\section{Facing biological risk in child and adolescence neuropsychiatric services during COVID-19 outbreak: Management of protection levels for the direct access}

\author{
Teresa Sebastiani ${ }^{1}$, Carlo Di Brina ${ }^{2}$, Maria L Tossali ${ }^{1 *}$, Morena \\ Tafuro $^{1}$ and Marco Marcelli ${ }^{1}$
}

${ }^{1}$ Primary Care Department, UOC TSMREE-DA, ASL of Viterbo, Italy

${ }^{2}$ Department of Human Neuroscience, Child and Adolescent Neuropsychiatry, Sapienza, University of Rome, Italy

\section{Editor}

COVID-19 emergency requests a new definition of security procedures adopted in Child and Adolescence Neuropsychiatric Services (CANS).

The WHO recommends using Personal Protective Equipment (PPE) for preventing inter-human transmission of viral diseases [1]. These recommendations regard primarily hospitalized patients and ambulatory medical services for adults.

In Italy, some attempts are emerging to prioritize rehabilitation activities in the context of inpatient rehabilitation settings [2].

Besides being simple and enabling the best protection, these procedures should avoid wasting resources, PPE over sizing and their misuse in particular.

Therefore, it was necessary to adapt these recommendations to the child and adolescent population in access to CANS, mainly focusing on the correct use of PPE and taking into account the typical variables of this population.

The age, the characteristics associated with the NeuroDevelopmental Disabilities, and the severity levels associated

\author{
More Information \\ *Address for Correspondence: \\ Dr. Maria Letizia Tossali, Mental Health Protection \\ and Development Rehabilitation Complex \\ Operative Unit, Adult Disabled (TSMREE-DA), \\ Local Health Authority Viterbo, Via E Fermi, 15, \\ 01100 Viterbo, Italy, \\ Email: marialetizia.tossali@asl.vt.it \\ Submitted: December 12, 2020 \\ Approved: December 28, 2020 \\ Published: December 29, 2020 \\ How to cite this article: Sebastiani $T$, \\ Di Brina C, Tossali ML, Tafuro T, Marcelli M. \\ Facing biological risk in child and adolescence \\ neuropsychiatric services during COVID-19 \\ outbreak: Management of protection levels for \\ the direct access. J Community Med Health \\ Solut. 2020; 1: 047-048. \\ DOI: 10.29328/journal.jcmhs. 1001006 \\ Copyright: () 2020 Sebastiani T, et al. This \\ is an open access article distributed under \\ the Creative Commons Attribution License, \\ which permits unrestricted use, distribution, \\ and reproduction in any medium, provided the \\ original work is properly cited. \\ Abbreviations: CANS: Child and Adolescence \\ Neuropsychiatric Services; PPE: Personal \\ Protective Equipment: RL: Risk Level

\section{W) Check for updates \\ OPEN ACCESS}

with the therapeutic needs can affect the correct adoption of these devices.

In this perspective, we experienced the definition of "Biological Risk" levels encountered in the patient's diagnostic and therapeutic direct approach.

We recruited 5 variables based on the patient's ability to tolerate PPE gear, the possibility of spreading the virus outside the barriers created by the PPE, and finally, the type of therapy needed.

The total score derived from the combination of the 5 variables levels ranges from 0 to 8 points and yields 6 levels of Biological Risk (from 0 to 5). Each risk level (RL) corresponds to well defined protection measures that health workers must adopt, namely a PPE gear and possible distancing measures. This scheme (Table 1) aims to improve the management of

Table 1: The five variables of Biological Risk.

\begin{tabular}{|c|c|c|c|c|c|}
\hline \multicolumn{1}{|c|}{ Variables } & & \multicolumn{3}{c}{ Points } \\
\hline A & Distancing [1] & Patient can keep distances & 0 & Patient cannot keep distances & 2 \\
\hline B & PPE gear [2] & Patient can wear PPE gear & 0 & Patient cannot wear PPE gear & 2 \\
\hline C & Parent's presence necessary in the room & Parent's presence not necessary & 0 & Parent's presence necessary & 1 \\
\hline D & drooling/ droplets/ incontinence (both urine and feces) & Not present & 0 & \\
\hline E & Repetition of touching (eyes, mouth, face) movements & Not present & 0 & \\
\hline
\end{tabular}


patients who arrive at the medical center/rehabilitation center, protect staff, and counteract the misuse of the security measures available (Table 1).

Thus, the RL derives from the summation of each variable's level. Bearing in mind available recommendations, we established a sort of Triage based on the treated patient's RL to define PPE gear and therapeutic setting organization.

In the case of RL $1 / 0$ (score range 0 to 1 , color of Triage green, Figure 1), the patient must wear a surgical mask and make frequent hand/glove sanitation. Health workers must wear a surgical mask and gloves. The treatment modality can be both individual or in pairs if the 2 children are RL $1 / 0$ or $0 / 0$.

In the case of RL 2/3 (score range 2 to 3, color of Triage yellow), the patient must wear a surgical mask and make frequent hand/glove sanitation. The health worker must wear Ffp2 mask, medical gown, single-use gloves, and visor mask to counteract drooling or droplets. The treatment modality is allowed in one to one only (Figure 1).
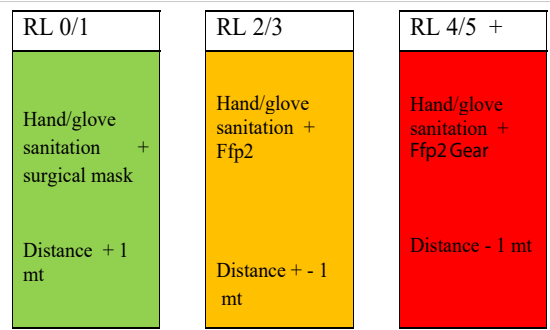

Figure 1: Recommendations for the correct use of the PPE, managing patients with different Risk Levels (RL).
In $\mathrm{RL} 4 / 5$ (score range 4 to 8 , color of Triage red), the patient must wear a surgical mask and gloves (or make hand sanitation), and it is desirable a patient training on how to wear the gear. The health worker must wear "Ffp2 Gear" (i.e., single-use gown, visor mask, gloves, medical cap, medical shoe cover, Ffp2 mask). The treatment modality is allowed in one to one only, in this case too.

In our opinion, the containment of the Sars-Cov-2 pandemic requires a precise quantitation of RL besides the disinfection measures [3]. A Triage relying on robust criteria ensures adequate protection to both the healthcare workers and patients and avoids wasting resources due to improper use of PPE gear.

\section{References}

1. World Health Organization. Rational use of personal protective equipment for coronavirus disease ([COVID-19)" and considerations during severe shortages: interim guidance|. WHO 2020.

https://apps.who.int/iris/handle/10665/331695

2. Boldrini P, Bernetti A, Fiore P. Impact of COVID-19 outbreak on rehabilitation services and Physical and Rehabilitation Medicine physicians' activities in Italy. An official document of the Italian PRM Society (SIMFER). Eur J Phys Rehabil Med. 2020; 56: 316-318. PubMed: https://pubmed.ncbi.nlm.nih.gov/32175719/

3. European Centre for Disease Prevention and Control Report Disinfection of environments in healthcare and non-healthcare settings potentially contaminated with SARS-CoV-2. ECDC 2020. https://www.ecdc.europa.eu/sites/default/files/documents/ Environmental-persistence-of-SARS_CoV_2-virus-Options-forcleaning2020-03-26_0.pdf 